\title{
Lésions iatrogènes dento-maxillaires en relation avec les actes pratiqués par les anesthésiologistes
}

\section{Iatrogenic dental and maxillary injuries associated with procedures performed by anesthesiologists}

\author{
Fabienne Jordana, DDS · Yvan Fronty, DDS · \\ Jacques Colat-Parros, DDS, PhD
}

Received: 19 December 2008/Revised: 3 March 2009/Accepted: 23 March 2009/Published online: 30 April 2009

(C) Canadian Anesthesiologists' Society 2009

\section{Au rédacteur en chef,}

Les traumatismes maxillo-dentaires qui surviennent à la suite du travail effectué par les anesthésiologistes engendrent un nombre important d'expertises en responsabilité médicale pour fractures dentaires, avulsions dentaires et fractures des réhabilitations prothétiques.

En France, un expert judiciaire désigné par le magistrat, en difficulté face à une question d'ordre médical, fournit les arguments de décision au juge. Cette désignation est appelée « mission ». Si l'expert conclut à une lésion iatrogène, les soins conservateurs ou prothétiques nécessaires à la réhabilitation du patient seront à la charge de l'assureur du praticien. Dans le cas contraire, les frais seront à la charge du patient. En cas de fractures dentaires peu importantes, des soins conservateurs sont nécessaires (restaurations au composite). Pour des fractures plus importantes, des prothèses scellées sont mises en place. En cas de fractures prothétiques, les prothèses sont renouvelées. En cas d'avulsion, une réhabilitation par prothèse conventionnelle ou par prothèse implanto-portée est réalisée.

Pour évaluer l'importance de la traumatologie dentomaxillaire liée aux actes des anesthésiologistes, nous avons adressé en 1999, puis en 2007, des questionnaires aux experts judiciaires français en odontologie ou en chirurgie

F. Jordana, DDS $(\bowtie) \cdot J$. Colat-Parros, DDS, PhD

UFR d'Odontologie, Université Bordeaux 2, Bordeaux, France

e-mail: jordana.fabienne@gmail.com

F. Jordana, DDS

Hôpital d'Instruction des Armées Robert Picqué, Villenave

d'Ornon, France

Y. Fronty, DDS

Hôpital d'Instruction des Armées Bégin, Saint Mandé, France maxillofaciale-stomatologie inscrits sur les listes des différentes juridictions françaises (303 experts en 1999 et 453 en 2007). Cette étude a été réalisée sous l'égide de l'université de Poitiers (France). Le taux de réponse a été de $42 \%$ en 1999 et de $31 \%$ en 2007.

Les questions posées aux experts étaient les suivantes :

- Êtes-vous missionnés pour des expertises relatives à l'anesthésie générale?

- En anesthésie générale, quels sont les principaux faits reprochés? Les réponses proposées sont : fracture dentaire ; avulsion intempestive de dents mobiles lors de l'intubation ou de l'extubation ; fracture prothétique ; autre.

La base de données a été générée sous Excel ${ }^{\circledR}$ (version 2007, Microsoft $\left.{ }^{\circledR}\right)$. Les tests du $\chi^{2}\left(\mathrm{Chi}^{2}\right)$ ont été réalisés avec les logiciels Epi-info ${ }^{\circledR}$ (version 6.04cfr, Organisation Mondiale de la Santé) et XIStats ${ }^{\circledR}$ (version 2007.5, Addinsoft $\left.{ }^{\circledR}\right)$.

En 1999, 10\% des missions des experts judiciaires français en odonto-stomatologie concernaient les traumatismes maxillo-dentaires provoqués par l'activité des anesthésiologistes. Cette proportion était de 9\% en 2007. En 1999, 39\% des experts judiciaires ont été sollicités au moins une fois pour ce type d'expertise; en 2007, ils ne sont plus que 34\%. En 1999 comme en 2007, les traumatismes maxillo-dentaires liés à l'activité des anesthésistes concernent principalement les fractures dentaires, les luxations dentaires et les fractures des reconstitutions prothétiques. En effet, en 1999, ces complications étaient rapportées respectivement par 70\%, 59\% et 59\% des experts missionnés pour des traumatismes attribués à l'activité d'un anesthésiste. En 2007, les chiffres n'avaient guère changé, avec $72 \%, 57 \%$ et $61 \%$, respectivement (Fig. 1). 


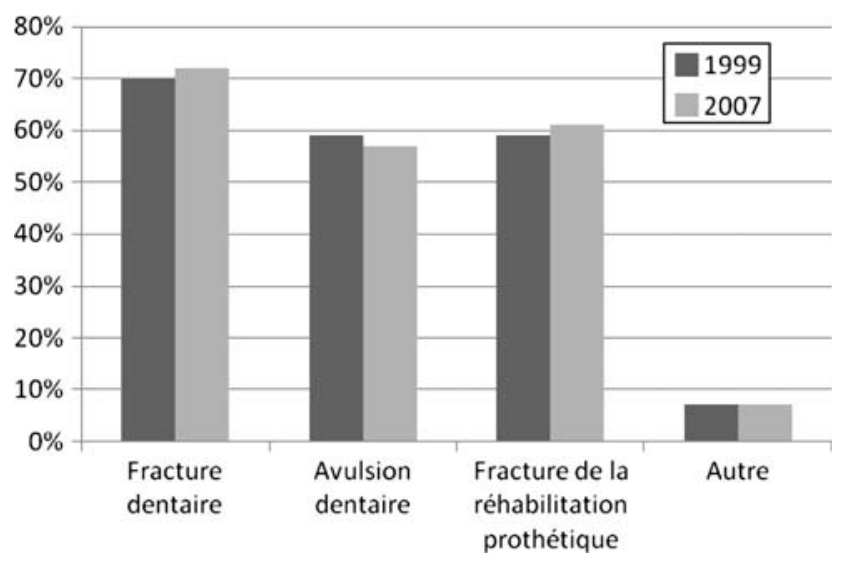

Fig. 1 Pourcentage des experts interrogés ayant été missionnés pour chacun des quatre types de traumatismes maxillo-dentaires liés aux actes des anesthésistes, en 1999 et en 2007

Lors de la consultation pré-anesthésique, des précautions devraient être plus particulièrement prises avant une intubation jugée difficile ou à risque. ${ }^{1}$ Toute visite préanesthésique devrait comporter une anamnèse dentaire et un examen rigoureux de la denture, en s'appuyant de préférence sur des protocoles standardisés. ${ }^{2}$ Dans le cadre du consentement éclairé, le patient devrait être informé du risque de traumatisme dentaire, en raison de la fréquence élevée des traumatismes liés aux actes des anesthésiologistes. Une visite de contrôle chez un dentiste avant une anesthésie avec intubation trachéale peut présenter un intérêt non négligeable. ${ }^{3}$ Chez les patients présentant un risque élevé au niveau du secteur dentaire antérieur, nous préconisons la réalisation d'un cliché radiographie rétroalvéolaire ou panoramique et d'une gouttière de protection en polymère. Ces actes préventifs pourraient certainement diminuer le nombre et le coût financier de traumatismes maxillo-dentaires iatrogènes dont sont reconnus responsables les anesthésiologistes.

Conflit d'intérêt Aucun.

\section{Références}

1. Clokie C, Metcalf I, Holland A. Dental trauma in anaesthesia. Can J Anaesth 1989; 36: 675-80.

2. Gatt SP, Aurisch J, Wong K. A standardized, uniform and universal dental chart for documenting state of dentition before anaesthesia. Anaesth Intensive Care 2001; 29: 48-50.

3. Chadwick $R G$, Lindsay SM. Dental injuries during general anaesthesia: can the dentist help the anaesthetist? Dent Update 1998; 25: 76-8. 